Mondes du Tourisme

du tourisme

$2 \mid 2010$

Vers une théorisation de l'approche géographique du tourisme

\title{
Performances touristiques
}

D'une métaphore à un cadre de pensée géographique renouvelé

\section{Amandine Chapuis}

\section{OpenEdition}

\section{Journals}

Édition électronique

URL : http://journals.openedition.org/tourisme/274

DOI : $10.4000 /$ tourisme. 274

ISSN : 2492-7503

Éditeur

Éditions touristiques européennes

Édition imprimée

Date de publication : 1 décembre 2010

Pagination : 44-56

ISSN : $2109-5671$

Référence électronique

Amandine Chapuis, «Performances touristiques », Mondes du Tourisme [En ligne], 2 | 2010, mis en ligne le 30 septembre 2015, consulté le 22 avril 2019. URL : http://journals.openedition.org/tourisme/274 ; DOI : 10.4000/tourisme.274

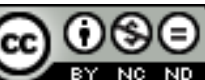

Mondes du tourisme est mis à disposition selon les termes de la licence Creative Commons Attribution - Pas d'Utilisation Commerciale - Pas de Modification 4.0 International. 


\title{
Performances touristiques
}

\section{D'une métaphore à un cadre de pensée}

\section{géographique renouvelé}

\author{
AMANDINE CHAPUIS_ [amandine.chapuis@gmail.com] \\ Doctorante en géographie, université Paris I - Panthéon-Sorbonne
}

Résumé. L'article propose une lecture géographique critique des travaux anglo-saxons qui se sont empa-

rés de la notion de performance, aujourd'hui toujours

quasiment absente de la littérature francophone. Le

champ des pratiques touristiques permet de questionner

les enjeux scientifiques de la notion en interrogeant ses origines et les courants de pensée qu'elle a engen-

drés. Désignant la pratique en situation d'un individu,

la performance permet d'appréhender l'incarnation,

la reproduction et la subversion des normes socio-

culturelles à travers la question de la performativité,

proposant une géographie sociale renouvelée des

phénomènes touristiques.
Abstract. The paper offers a critical reading of anglo-saxon literature on the notion of performance, from a geographical perspective. The field of the tourist practices allows to question the scientific issues of the notion (its origins and developments), which remains almost unexploited in the French-speaking literature. Standing for an individual practice in situation, the performance allows to apprehend the embodiment, reproduction and subversion of socio-cultural norms, through the issue of performativity, thus proposing a renewed social geography of tourist phenomenons. 
L

a métaphore artistique de

la performance est loin

d'être la seule manière d'appréhender les pratiques individuelles, mais elle a pris une ampleur assez importante en sciences sociales pour que certains auteurs parlent désormais de performative turn (voir, en anthropologie par exemple, Conquergood, 1989) ou que des volumes spéciaux de revues scientifiques internationales lui soient consacrés (voir, en géographie, Thrift, Dewsbury, 2000). À l'origine simple analogie, elle est maintenant devenue une notion liée au champ de la performativité, proposant un cadre de pensée théorique qui permet de renouveler une approche géographique des pratiques, notamment touristiques (c'est le champ qui sera abordé ici). Si la géographie anglo-saxonne s'est clairement emparée de la performance depuis les années 1990 (voir notamment Bell, Binnie, Cream, Valentine, 1994 ou Crang, 1994), elle reste encore quasiment absente de la littérature francophone, ce dont témoigne la recherche bibliographique.

Ce texte a donc pour objectif de présenter une approche critique des travaux anglo-saxons sur la performance, qui permette à la géographie francophone de mieux s'en emparer (Chivallon, 1999). Il propose un point théorique sur cette notion, en interrogeant les courants de pensée qu'elle a engendrés, en géographie et, de manière complémentaire, dans les tourism studies (domaine ouvert à d'autres sciences sociales, voir par exemple Coleman, Crang, 2002a ; Baerenholdt, Haldrup, Larsen, Urry, 2004). Il envisage l'analyse des pratiques touristiques comme un champ privilégié pour questionner les enjeux scientifiques de la notion de performance.

Le terme "performance" ne sera pas employé dans le sens courant d'efficacité, comme c'est le cas dans les travaux de l'Équipe Mit, par exemple, pour désigner la performance que constitue pour les lieux touristiques le fait de durer dans le temps (Knafou, 1996). Il ne s'agit pas non plus de l'entendre au sens d'un acte artistique exceptionnel, comme on emploie aujourd'hui en France le terme "performeur". Son origine est à rechercher dans la citation de Shakespeare "All the world's a stage" (dans As you like it, 1623); le monde est une scène de théâtre, les actions humaines sont des rôles joués par les individus. On emprunte donc aux études artistiques ce terme de langue anglaise (Schechner, 2002). Dans les départements de performing arts, il signifie le fait d'accomplir une représentation artistique (une pièce de théâtre, un opéra, un ballet, par exemple). Il désigne à la fois l'exécution de techniques artistiques acquises et l'interprétation de thèmes imposés, voire parfois la capacité d'improvisation des artistes. Il n'existe pas en français de mot exprimant cette combinaison d'idées, à l'exception sans doute de "représentation", qui, dans la logique d'une métaphore artistique, semble pertinent. C'est d'ailleurs le choix d'Alain Accardo, le traducteur en français d'un des deux principaux auteurs de la performance, Erving Goffman, aux éditions de Minuit pour The Presentation of self in every day life (Goffman, 1973). Toutefois, dans une perspective disciplinaire géographique, l'emploi du terme "représentation" est gênant, car il correspond à l'idée de construction mentale de l'image d'un lieu, d'un territoire ou de tout autre réalité spatiale. Les représentations spatiales ont été particulièrement étudiées par les courants de géographie culturelle et humaniste, or, précisément, certains des auteurs qui se réfèrent aujourd'hui à la notion de performance critiquent les perspectives humanistes $^{(1)}($ Nash, 2000). L'approche non représentationnelle, prônée notamment par Nigel Thrift, oppose quasiment performance et représentation, la représentation étant une image, une description du monde, ayant une dimension essentiellement intellectuelle, alors que la performance désigne un corps en action, qui produit perpétuellement une nouvelle réalité (Thrift, 2000b). Cynthia Kraus, la traductrice pour les éditions La Découverte de l'ouvrage de Judith Butler (deuxième auteur fondamental sur cette question) Gender Trouble, prend le parti de ne pas traduire le terme et même de le faire passer directement dans la langue française, sans italique, sans guillemets (Butler, 2005). Êtant donné que les termes "performatif" et "performativité" (issus de la 
linguistique) existent désormais en français et que le travail de Butler s'inspire largement de la speech act theory ${ }^{(2)}$, cette option semble la plus pertinente. Elle a le mérite de venir compléter le champ lexical de la performativité, de plus en plus employé en géographie.

La géographie s'est en effet progressivement emparée de la métaphore de la performance (Thrift et Dewsbury, 2000), en tant qu'objet (Nash, 2000), en tant que simple analogie (Crang, 1994), ou en tant qu'outil conceptuel (Longhurst, 2000). Ce triple emploi, assignant des statuts divers au terme, rend particulièrement complexe la rédaction d'une définition univoque. S'ajoute à cette difficulté celle de la double filiation de la notion, les auteurs l'ayant utilisée au cours des vingt dernières années se référant principalement soit à Erving Goffman, soit à Judith Butler, deux auteurs l'ayant largement développée, mais dans des perspectives significativement différentes. Néanmoins, il est possible, pour débuter la réflexion, de s'accorder sur le fait que la performance désigne la pratique en situation d'un individu, en ce qu'elle incarne des normes socioculturelles qui la régissent mais aussi en ce qu'elle participe ellemême à la reproduction et/ou à la subversion de ces normes.

Cet article propose donc un état des lieux sur la performance, en interrogeant ses filiations et les approches qu'elle a engendrées. Si la progression de la métaphore et l'émergence du cadre théorique qui en découle seront appréhendées principalement du point de vue de la géographie, il ne faut pas oublier qu'il s'agit d'un mouvement qui touche l'ensemble des sciences sociales, ce que la réflexion sur le champ du tourisme permettra de souligner. On pourrait même dire que les circulations réalisées par le terme entre disciplines témoignent d'une reconfiguration à l'œuvre dans le champ des sciences sociales, autour de perspectives épistémologiques et d'objets d'étude. Nous verrons ainsi en quoi l'ensemble théorique qui se dessine autour de la notion de performance peut permettre de renouveler l'appréhension des phénomènes touristiques.

\section{VERS UN PERFORMATIVE TURN?}

Pour Nigel Thrift, la performance est actuellement une des métaphores les plus envahissantes ${ }^{(3)}$ des sciences humaines (Thrift, 2000b). L'analyse de la littérature ${ }^{(4)}$ permet de mettre en évidence l'apparition du terme dans le vocabulaire scientifique et le développement de l'utilisation de cette analogie comme cadre théorique, tant en géographie que dans les études consacrées aux phénomènes touristiques.

\section{La montée en puissance de la performance}

En géographie, c'est dans les années 1990 que la performance fait son apparition dans deux types de débat. D'une part, les auteurs participant au tournant culturel en géographie économique s'y intéressent. Ils remarquent que, dans une économie tertiarisée, l'analogie avec le domaine artistique fonctionne assez bien pour décrire le comportement des employés des entreprises de services. Philip Crang étudie, par exemple, les relations sociospatiales de consommation à travers l'exemple d'un restaurant populaire en Angleterre. Il analyse les pratiques professionnelles des serveurs comme des performances et décrit la géographie qu'elles dessinent à l'intérieur du restaurant (Crang, 1994). D'autre part, le terme apparaît à la faveur du développement des gender studies en géographie, ce dont témoigne le débat qui a alors lieu dans la revue Gender, Place and Culture. David Bell, Jon Binnie, Julia Cream et Gill Valentine sont parmi les premiers à l'importer, dans un sens non plus métaphorique mais notionnel. Dans un article commun, ils étudient la perturbation des mécanismes de production du caractère hétérosexuel de l'espace public à travers les pratiques de deux groupes, les gays skinheads (gays à l'allure très virile) et les lipstick lesbians (lesbiennes à l'allure très féminine) (Bell, Binnie, Cream et Valentine, 1994). Au début des années 2000, l'emploi du terme se généralise et devient l'objet de débats. Nigel Thrift établit un lien direct entre la performance (en tant que notion permettant de désigner des pratiques sociales) et les théo- 
ries non représentationnelles : la performance, s'intéressant aux corps en action, doit être l'objet favori d'une approche qui privilégie les pratiques par rapport aux représentations (Thrift, 2000b). L'auteur coordonne, avec John-David Dewsbury, un numéro thématique de Environment and Planning D, intitulé "Space and performance" (Thrift et Dewsbury, 2000). Ils prennent acte de la diversité des filiations observables dans les travaux géographiques s'intéressant à la performance. Mais ils insistent sur ce qu'ils considèrent comme leur apport majeur : celui de mettre les pratiques, et non plus les discours, au centre de la réflexion géographique. Pour Thrift et Dewsbury, l'ensemble de ces travaux partagent un intérêt pour l' "incarnation" ("embodiment") de la géographie et cherchent à la "réanimer" ("animate new") : "above all, we argue, they want to make space livelier. They want to produce spaces which flirt and flout, gyre and gimble, twist and shout" [Nous soutenons avant tout qu'ils veulent animer l'espace. Ils veulent produire des espaces qui batifolent et se moquent, qui font la ronde et s'emmêlent, zigzaguent et crient]. Par la suite, le vocabulaire et le cadre théorique de la performance occupent une place majeure dans cette revue (trois articles en 2003 et 2006, quatre en 2002, cinq en 2008 et six en 2007). Cette notion s'est diffusée à d'autres publications comme Progress in Human
Geography (six articles entre 1996 et 2002, deux en 2005, 2006 et 2007, quatre en 2004, cinq en 2003 et jusqu'à onze en 2008), par exemple. Elle traverse également des domaines variés de la discipline, de la géographie post-coloniale (Hoelscher, 2003 ; Pannelli, 2008) à la géographie urbaine (Lees, 2002; Latham et McCormack, 2004; Jayne, Valentine et Holloway, 2008), en passant par la géographie rurale (Little et Leyshon, 2003), politique (Sparke, 2008), celle des inégalités sociales (Holt, 2008), des religions (Bailey, Harvey et Brace, 2007), des migrations (McDowell, 2008) ou même la réflexion cartographique (Perkins, 2004 ; Crampton, 2009).

Dans les études touristiques, la métaphore de la performance a été employée de manière plus précoce, parce que le principe de définir le tourisme comme un jeu (pas seulement au sens ludique, bien sûr) et de définir les pratiques, notamment des guides touristiques ou des communautés locales, comme des rôles d'acteurs, est une idée assez commune, portée notamment par l'anthropologie (Coleman et Crang, 2002b ; Chaney, 2002). C'est avant tout Dean MacCannell, s'inspirant des travaux d'Erving Goffman, qui a largement contribué à diffuser une conception métaphoriquement théâtrale des phénomènes touristiques, à travers ses questionnements sur la mise en scène de la culture. La réalité dévoilée au touriste serait une "staged authenticity", une culture mise en scène, que l'on donne à voir aux visiteurs (MacCannell, 1999). Cette conception a profondément influencé l'ensemble des travaux ultérieurs en tourism studies.

Tout d'abord, la performance désigne donc la manière dont travailleurs du tourisme et populations locales (guides, danseurs folkloriques, acteurs, artisans, commerçants et autres charmeurs de serpents) adoptent des rôles prédéterminés dans le but de présenter une certaine image de leur culture (cf., par exemple : sur les employés de l'industrie des croisières, Weaver, 2005 ; sur la mise en tourisme de la société dogon, Doquet, 2002). Il s'agit alors d'appréhender la question de la production des identités locales à travers le tourisme. Cependant, la performance tend de plus en plus à désigner les pratiques des touristes en insistant sur la reproduction de normes sociales (Coleman et Crang, 2002a). La thèse de sociologie spatiale de Tim Edensor, Tourists at the Taj, performance and meaning at a symbolic site, publiée en 1998, va dans ce sens : les pratiques spatiales des touristes autour du Taj Mahal sont appréhendées comme des performances, des chorégraphies codifiées, dont la répétition dans le temps produit des normes de comportement touristique (Edensor, 1998).

La performance est aujourd'hui présente dans un grand nombre de publications sur le tourisme. Parfois, le terme est simplement un syno- 
nyme modernisé de "pratique de consommation" (Baerenholdt, Haldrup et Larsen, 2004); dans ce cas, son emploi ne semble pas se justifier. En revanche, il apparaît plus légitime lorsqu'il participe à la construction d'une approche renouvelée des pratiques touristiques autour de la question de la performativité. C'est le cas d'un ouvrage coordonné par Claudio Minca et Tim Oakes (Minca et Oakes, 2006a), où la majorité des articles se réfère à la performance (Edensor, 2006 ; Crang, 2006 ; Jacobs, 2006 ; Minca, 2006; Oakes, 2006).

Jessica Jacobs analyse par exemple la manière dont des femmes occidentales font l'expérience de rencontres sexuelles tarifées avec des hommes égyptiens dans les stations balnéaires du Sinai (Jacobs, 2006). C'est la performance (la mise en œuvre) de leur condition féminine occidentale moderne qui rend possible, dans le temps et le lieu spécifiques et bien délimités de la pratique touristique, l'adoption de pratiques considérées comme éminemment masculines. Dans sa thèse, Rachele Borghi explique comment la place Jamaa El Fna à Marrakech est un lieu touristique sans cesse coproduit par les performances des guides, artisans et commerçants, en interaction avec celles des touristes, dans un cadre mis en scène par la patrimonialisation depuis le protectorat français et l'action de Lyautey (Borghi, 2006). C'est l'ensemble des conditions de production des interac- tions sociales dans le cadre des situations touristiques qui peut ainsi être pensé à travers le cadre de la performance (voir aussi Minca, 2009).

\section{Double filiation théorique : de la métaphore au cadre de pensée de la performativité}

Plusieurs articles anglophones ont fait le point sur les différentes approches de la performance en géographie (Thrift, 2000b ; Gregson et Rose, 2000 ; Pratt, 2009). Tous mettent en évidence la prédominance de deux conceptions : l'analogie entre performances et interactions sociales spatialisées, influencée par le travail d'Ervin Goffman, d'une part, et la performance comme origine et expression de la performativité, approche issue du travail de Judith Butler, d'autre part.

Dans La Mise en scène de la vie quotidienne, Goffman adopte la perspective de la représentation théâtrale (Goffman, 1973). Au quotidien, les individus "acteurs" endossent des "rôles" qu'ils adaptent en fonction de la "scène" (le lieu) et $\mathrm{du}$ "public" (les autres individus présents). Ces rôles sont prédéterminés socialement. Cette conception implique une typologie des espaces de vie au sein desquels l'individu est plus ou moins en représentation. Il établit ainsi une distinction entre front régions (celles de la performance) et back regions (celles du repos, où l'on peut être "soi-même"). Crang réinvestit directement cette idée en géographie de la consommation, dans l'article évoqué précédemment (Crang, 1994). Les serveurs du restaurant dont il observe pendant plusieurs mois les pratiques professionnelles (en travaillant lui-même comme serveur au sein du restaurant), transforment temporairement leur manière d'être pour s'adapter au comportement attendu par leurs clients. Ils endossent ce rôle dans les front regions du restaurant (principalement la salle) et relâchent cette contrainte dans les back regions (l'office, la réserve, la cuisine). MacCannell adopte la même perspective.

Si Judith Butler emploie elle aussi le terme "performance", l'analogie théâtrale occupe une place marginale dans son travail, qui a pour sujet le genre et la sexualité. Dans Trouble dans le genre, le féminisme et la subversion de l'identité, elle montre que non seulement le genre est une construction sociale, mais qu'il est le produit des pratiques quotidiennes (performances) des individus (pas seulement de discours prescriptifs ou d'une histoire symbolique) et qu'il est un processus sans fin (Butler, 2005). Pour elle, à l'inverse de ce que propose Goffman, il n'existe pas d'identité fixe, que l'on mettrait de côté pour endosser des rôles prédéterminés dans différentes situations, mais l'identité est une construction permanente à travers les pratiques. C’est donc la répétition des performances dans le temps qui produit les normes sociales auxquelles 
ces performances adhèrent ou qu'elles viennent au contraire réfuter. Butler s'inspire de la speech act theory des linguistes, qui pose que les énoncés ne sont pas seulement des descriptions de la réalité mais qu'ils agissent sur elle (Austin, 1970). Pour la philosophe, non seulement les discours, mais aussi les pratiques participent de ce processus performatif. Pourtant, elle ne rejette pas l'analogie théâtrale, qui est même à la base de son raisonnement : on joue le rôle de la femme ou de l'homme. Simplement, elle réfute l'idée d'une dualité de l'identité : ces rôles que l'on endosse plus ou moins consciemment produisent bel et bien ce que nous sommes et on ne peut s'en défaire comme de vulgaires enveloppes. Le jeu est donc beaucoup plus complexe qu'il n'y paraît, il est une mise en cause constante de l'identité, une production toujours inachevée.

Si l'on transpose cette idée en géographie sociale, comme l'ont fait Bell, Binnie, Cream et Valentine dans l'article précédemment évoqué, on peut davantage penser l'espace comme coproduit par les pratiques (Bell, Binnie, Cream et Valentine, 1994). Les auteurs cherchent, en effet, à montrer que le caractère hétérosexuel des lieux est autant le fruit d'une production sociale que leur caractère homosexuel (ce qui est plus couramment admis, puisque la norme sociale hétérosexuelle dominante considère les lieux homosexuels comme "anormaux"). Ils analysent la manière dont les pratiques des gays skinheads et des lipstick lesbians dans l'espace public viennent troubler l'ordre spatial et révéler le caractère construit de l'hétérosexualité des lieux.

Un des enjeux les plus importants pour la compréhension de ces deux approches est la question de leur positionnement dans le débat structure / agency. Quelle place accordent-elles au libre arbitre des acteurs ? À la première lecture, la marge de manœuvre des individus semble globalement réduite. Leur intentionnalité n'est un sujet de discussion central ni pour Goffman, ni pour Butler. Dans les deux cas, la répétition des performances mène au renforcement des normes et structures sociales. Pourtant, les deux auteurs sont plus nuancés. Goffman prend en compte une intentionnalité stratégique des acteurs, orientée vers un but unique, celui de donner une certaine image de soi. La marge de manœuvre des individus est contrainte par la nécessité de la réalisation d'une posture sociale. En ce qui concerne Butler, c'est encore plus complexe, car, si on lui a parfois fait remarquer que la manière dont elle pense la performance est proche de la manière dont Bourdieu envisage l'habitus (donc par la répétition, laissant peu de place à l'intentionnalité), on lui a également reproché, au contraire, de faire du genre un simple choix individuel. Judith Butler s'est donc efforcée de montrer que le genre, s'il peut être un jeu au sens goffmanien, n'est pas un "simple" choix, mais qu'il est bien davantage contraint, elle accepte d'ailleurs volontiers dans ce sens le rapprochement avec Bourdieu. Dans le même temps, elle insiste sur le caractère subversif de certaines performances, capables de faire évoluer la norme.

Nigel Thrift, qui importe directement la notion de performance dans ses efforts pour mettre en place le cadre des théories non représentationnelles, semble faire figure d'exception. En effet, il ne s'appuie d'emblée ni sur Goffman, ni sur Butler, mais fait référence davantage à Michel de Certeau (Thrift, 2000b). Il insiste en effet sur les pratiques en train de se faire, sur les manières de faire des individus avec les lieux : "performance is the art of producing the now, a means of theorizing the day-to-day improvisations which are the means by which the now is produced" [La performance est l'art de produire le présent, un moyen de théoriser les improvisations quotidiennes qui sont le moyen par lequel le présent est produit] (Thrift, 2000a ; de Certeau, 1990). C'est la matérialité corporelle et le caractère préréflexif des pratiques qu'il interroge et non les représentations, la vie intérieure du sujet. Pour développer cette approche, il s'inspire du domaine de la danse qu'il conçoit comme une pratique préréflexive au cours de laquelle quasiment seul le corps compte. 
On peut évidemment lui reprocher un déni très contestable des dimensions symbolique, genrée et politique de la danse, mais ce qui l'intéresse pour asseoir la performance comme socle de l'approche non représentationnelle, c'est cette primauté de la corporéité. Thrift insiste, beaucoup plus que Goffman ou Butler, sur les possibilités d'improvisation des individus, il laisse la place à leur créativité. Mais, en réalité, ces innovations ne sont pas, selon lui, le fruit des intentionnalités multiples des acteurs, mais celui de la contingence qui découle de la présence physique des corps en situation, des arts de faire avec les lieux : "arising especially out of the expressive powers of the body, these improvisations enable each moment to be open to possibility" [ces improvisations, qui surgissent notamment des pouvoirs expressifs du corps, permettent à chaque moment d'ouvrir des possibles] (Thrift, 2000a).

\section{POUR \\ UNE GÉOGRAPHIE SOCIALE CRITIQUE DES PRATIQUES TOURISTIQUES}

Pour David Chaney, "from an appreciation that tourist attractions are representations that appeal to different audiences in a variety of ways, indicating in this phrasing that they are in important respects designed constructions, its seems to me natural therefore that the dominant metaphor in tourism studies has been the idea that the tourist setting should be imagined as a form of performance or staging" [Partant $\mathrm{du}$ fait que les attractions touristiques sont des représentations qui parlent aux différents publics de multiples façons, c'est-à-dire qu'elles sont à de nombreux égards des constructions voulues, il m'est apparu naturel que la métaphore principale dans les tourism studies était l'idée que la posture du touriste devait être conçue comme une forme de performance ou de mise en scène] (Chaney, 2002, p. 196). Comment la géographie peut-elle s'inspirer de cette métaphore de la performance pour construire un cadre théorique qui renouvellerait l'appréhension des pratiques en général et des pratiques touristiques en particulier?

\section{Penser les processus \\ de production des performances et des lieux touristiques}

Le cadre de la performativité permet de repenser la question des normes et de la liberté des acteurs en matière de pratiques touristiques et de production de lieux touristiques. Dans quelle mesure les touristes sont-ils tributaires des discours normés et prescriptifs de l'industrie touristique et des guides de voyages ? Comment les encadrements touristiques façonnentils pratiques et imaginaires ?

Un certain nombre de travaux insistent sur le caractère codifié des pratiques touristiques. L'idée qu'on ne naît pas touriste, mais qu'on le devient, en acquérant un certain nombre de compétences et en intériorisant des codes, est illustrée par Orvar Löfgren : il s'attache en particulier à déconstruire les discours et les images (à travers les journaux, les récits de voyages, les publicités, entre autres) qui orientent la manière dont on devient touriste, en Occident (Löfgren, 1999). Si on peut, à partir de ce type de travaux, étudier le caractère performatif de ces discours, le rôle des pratiques en elles-mêmes n'est pas souligné par Löfgren. Tim Edensor, lui, a abordé cette problématique en appréhendant les pratiques des touristes au Taj Mahal comme des performances (Edensor, 1998). Ses textes insistent sur la reproduction de pratiques normées. Pour lui, même des expériences ayant pour but de s'opposer aux normes traditionnelles établissent en fait un autre univers codifié où les pratiques répondent également à des règles relativement strictes. C'est en particulier le cas des backpackers.

Cette question de la liberté d'action des individus dans leurs pratiques et de leur marge de manœuvre vis-à-vis des cadres est également liée à la réflexion sur les rapports entre pratiques et lieux touristiques. Déjà chez Goffman, les lieux sont des décors pour l'action, des contextes, mais des contextes qui influencent le type de performance des individus (plus ou moins contraints) (Goffman, 1973). Dans ce débat sur le poids des encadrements, Edensor insiste 
sur le fait que les lieux touristiques sont aménagés justement pour accentuer le processus performatif, contrôler les pratiques et minimiser les possibilités d'improvisation de la part des touristes (Edensor, 200 I). Le comptoir touristique, par exemple, serait pour lui un enclavic tourist space (espace touristique enclavé), où les possibilités d'improvisation par rapport à la norme prescrite seraient des plus réduites. Vincent Coëffé suggère une analyse significativement différente en insistant au contraire sur la forte densité des comptoirs qui maximise les interactions humaines possibles et donc la contingence des rencontres (Coëffé, 20l0). Cependant, on pourrait peut-être trancher cette question en prenant en compte la diversité des modalités d'encadrement des pratiques, en premier lieu corporelles, dans certains comptoirs touristiques, qui mène Claudio Minca à penser ces lieux comme des expressions totalitaires modernes (Minca, 2009) $)^{(5)}$.

Cette réflexion sur les liens entre lieux et pratiques touristiques trouve ses racines chez MacCannell (directement inspiré par le travail de Goffman) avec l'idée des front et back regions : les touristes n'ont accès qu'au front stage (le devant de la scène) dessiné par les sociétés locales, tandis qu'elles préservent des back stages que les touristes, conscients de la mise en scène et disposant d'un regard critique, cherchent à atteindre, dans leur quête d'authenticité (MacCannell,
200 I). Il semble que cette approche, dont la dimension spatiale reste métaphorique chez MacCannell (les front stage et back stage sont des espaces sociaux), pourrait être utilement transposée par les géographes pour comprendre la spatialisation des performances touristiques, en particulier dans les métropoles $^{(6)}$. Une hypothèse serait que les front stages du tourisme correspondent aux hyper-centres touristiques, "patrimonialisés", "festivalisés", ou aux comptoirs périphériques intégrés (par exemple les parcs d'attraction). Là se concentreraient les efforts de mise en scène du paysage urbain à des fins touristiques: esthétisation, "exotisation” (Staszak, 2008), production d'un espace de consommation culturelle capable d'accueillir les pratiques les plus encadrées par l'industrie touristique. À l'inverse, les back stages, lieux moins centraux et moins directement mis en scène, occuperaient des rôles de séduction alternative, propices à des pratiques distinctives, car effectivement accessibles seulement aux touristes les plus autonomes et expérimentés, ou introduits.

L'accès au back stage, correspondant à une émancipation visà-vis des structures et des formes les plus strictes de reproduction des normes, pourrait être pensé comme la valorisation d'une forme de capital spatial et/ou touristique accumulé par l'intermédiaire de performances quotidiennes et/ou touristiques antérieures. Il s'agirait d'une situation où le processus performatif deviendrait la condition, non pas de la reproduction des normes, mais du dépassement de ces normes. Cependant, en aucun cas cette distinction spatiale ne doit être essentialisée. D'abord, parce que l'authenticité d'un back stage non mis en scène constitue une pure idéalisation. Ensuite, parce que l'approche par les performances des touristes permet de comprendre que, du point de vue des individus, la distinction front stage/back stage représente une limite mouvante (que l'on pourrait qualifier en termes de capitaux économiques, mais surtout culturels et sociaux) ou, plus encore, une sorte de front pionnier personnel imaginé, dont chacun joue tout au long de son voyage $\mathrm{e}^{(7)}$.

Une proposition de Claudio Minca et Tim Oakes va dans le même sens, c'est ce qu'ils appellent le "paradoxe des voyages" (Minca et Oakes, 2006b). Ils partent du principe que le caractère performatif des pratiques touristiques (dans le sens de répétition de pratiques normées) offre la possibilité d'avoir des pratiques touristiques non réflexives, ce qui est reposant, tant mentalement que physiquement. Dans le même temps cependant, le tourisme offre tout un tas de situations propices à la réflexivité, ne serait-ce que par le déplacement hors du contexte de vie quotidien et par le relâchement des contraintes sociales qui en découle. Si l'on poursuit ce raisonnement, ce serait en fonction de l'intentionnalité du tou- 
riste, de son projet re-créatif ${ }^{(8)}$, que le processus performatif mènerait soit à la reproduction, soit à l'innovation.

Le cadre théorique de la performativité permet donc d'interroger les processus de construction de la manière dont on pratique touristiquement les lieux. L'attention portée aux processus plutôt qu'aux états constitue ainsi un apport fondamental de la notion de performance : "instead of seeing places as relatively fixed entities, to be juxtaposed in analytical terms with more dynamic flows of tourists, images and cultures, we need to see them as fluid and created through performances" [Au lieu de considérer les lieux comme des entités relativement figées, qui sont juxtaposées de manière analytique avec des flux dynamiques de touristes, d'images, de cultures, il nous faut les considérer comme variables et créés par les performances] (Coleman et Crang, 2002b, p. I). À travers le processus performatif, on peut penser la construction des normes touristiques précisément par la répétition de performances, et pas seulement comme une réponse collective à des discours prescriptifs. De la même manière, les lieux apparaissent effectivement comme coconstruits par les performances des différents acteurs (Borghi, 2006). Il s'agit de se départir définitivement d'une conception essentialiste des lieux, d'une identité spatiale qui serait une donnée figée, perturbée par l'arrivée du tourisme.
Au contraire, les qualités des lieux touristiques sont produites par les performances répétées ou innovantes des touristes, autant que par les stratégies des acteurs locaux. La compréhension du caractère performatif des pratiques spatiales permet ainsi de renouveler la question de la production sociale de l'espace.

\section{Performances touristiques, corporéités et construction des identités spatiales}

Pour Judith Butler comme pour Nigel Thrift, c'est le corps qui constitue l'élément central de la réflexion sur la performance. La question de la corporéité dans le champ du tourisme peut également être repensée en s'appuyant sur ce cadre. Jusqu'à présent, elle a surtout été réduite à celle des sens. En effet, en réponse au "tourist gaze" de John Urry (Urry, 1990), un certain nombre de travaux se sont appliqués à montrer que la vue n'est pas le seul sens impliqué dans l'expérience touristique (Bell et Lyall, 2002 ; Crouch et Desforges, 2003). Le tourisme comme expérience multisensorielle, abordée à travers des terrains variés comme l'hiver finlandais (Nielsen, 2003), le Grand Canyon (Neumann, 2002), les stations touristiques de Palmanova et Magaluf aux Baléares (Andrews, 2005), ou des pratiques comme la spéléologie (Cant, 2003), peut alors être compris comme une quête de plaisir physique. Sentir la chaleur du soleil sur sa peau, la force des vagues sur ses jambes ou le goût du sel sur ses mains peut constituer un moteur de la pratique touristique, une sensation imaginée toute l'année, qui se concrétise enfin au moment des vacances... Dans cette perspective, on remarque que c'est bien la performance, la présence physique, la rencontre avec des lieux aux qualités singulières, qui est au cœur de l'expérience touristique. Il n'y a pas d'intermédiaire possible, de virtualisation envisageable ; la médiation, quelle qu'elle soit, ne peut avoir pour enjeu que la sublimation par une forme de narration (photo, vidéo, journal ou autre) de l'expérience vécue, qui elle, prime.

Par ailleurs, les corps touristiques ont pu être considérés comme des révélateurs de normes sociales. Löfgren prend l'exemple d'une carte postale des années 1950 intitulée "By the beautiful sea", qui met en scène des touristes américains montrant par l'exemple quelles postures corporelles sont les plus appropriées sur la plage (Löfgren, 1999). Il souligne ainsi la manière dont les corps sont les premières expressions des codes normatifs liés au tourisme balnéaire à ce moment et dans ce lieu.

Mais le travail de Butler permet de montrer que les postures des corps ne sont pas seulement l'expression de normes, ils ne font pas que révéler, ils créent également. Dans Gender Trouble, elle remet en cause la distinction sexe (naturel, fixe) / genre (construit socialement) : le sexe (l'illusion d'une 
appartenance biologique) est tout autant construit que le genre. Face à l'accusation de négation de la matérialité du corps, elle montre au contraire que c'est cette matérialité qui produit sexes et genres. "Le genre consiste en une identité tissée avec le temps par des fils ténus, posée dans un espace extérieur par une répétition stylisée d'actes" (Butler, 2005, p. 265). Le genre est donc produit par la manière dont toutes sortes de gestes, de mouvements corporels donnent l'illusion d'un soi genré. Le sexe devient alors une fiction sociale, l'effet de la sédimentation, de l'incorporation au sens fort, des normes de genre. Alors que tout ce qui se passe à la surface du corps semble refléter une "essence” de l'identité, ce sont en fait ces performances qui produisent la "fiction genrée". On peut ainsi imaginer étendre ce raisonnement pour comprendre la manière dont les normes de comportement touristique sont produites par la répétition de pratiques dont la corporéité serait la dimension essentielle. Il semble que ce soit la direction dans laquelle s'engage Vincent Coëffé (bien qu'il n'utilise pas la notion de performance) lorsqu'il met l'observation des corps au centre de sa démonstration de la manière dont les pratiques touristiques produisent l'urbanité des lieux (Coëffé, 2007).

La poursuite de cette réflexion conduit à appréhender le temps touristique comme un moment à part entière de construction de l'identité individuelle. Cette perspective a connu un certain nombre de développements qui se sont efforcés de montrer les imbrications, pour les individus, entre temps touristique et temps du quotidien. Il peut s'agir, par exemple, des enjeux biographiques, initiatiques du voyage (Tucker, 2005) ou bien de la construction familiale (Haldrup et Larsen, 2003). En revanche, la dimension spatiale de l'identité a peu été abordée. L'idée que les lieux de résidence ne sont pas les seuls à avoir du sens dans le système de mobilité des individus, mais que ceux du tourisme peuvent également avoir des significations importantes a été défendue par Mathis Stock (Stock, 200I).

Or, on pourrait aller plus loin en faisant l'hypothèse que le tourisme, favorisant la confrontation avec des lieux non quotidiens, constitue un moment privilégié de la mise en œuvre (performance) du rapport des individus à l'espace et aux lieux. Par exemple, la pratique touristique des espaces urbains, en particulier ceux des métropoles mondiales, nécessite l'utilisation de compétences spatiales spécifiques, que l'on acquiert au quotidien et/ou au cours de précédentes expériences touristiques. Elle est rendue possible par la mobilisation d'une forme de rapport à l'urbanité, qui s'exprime dans les arts de faire des touristes avec les lieux, dans la manière dont ils construisent les espaces urbains comme les paysages touristiques, dans la façon dont ils les lisent et les investissent, en lien avec leurs projets re-créatifs. On peut penser que ces performances touristiques ne se contentent pas de mobiliser mais qu'elles nourrissent, font évoluer, produisent cette dimension spatiale de l'identité individuelle, selon un processus performatif qui serait, comme le propose Butler, actif à l'échelle de l'individu et non pas seulement à l'échelle des structures sociales $^{(9)}$.

Cette perspective d'interrogation de la dimension spatiale de la production de l'identité des individus constitue un apport critique de la notion de performance. Elle offre des orientations stimulantes à une géographie qui place l'échelle des individus au cœur de ses questionnements, en même temps qu'elle permet de repenser la question, essentielle pour la discipline, des rapports des individus aux lieux. Aux côtés des géographies culturelles et phénoménologiques, elle propose des pistes pour envisager une approche sociale, qui contient en germe un lien fort entre individus et sociétés (par le biais de la réflexion sur les pouvoirs et les normes, impliquée par le cadre théorique de la performativité).

\section{Réflexions finales}

Tandis que l'emploi de la performance se diffusait, on a quasiment cessé de s'interroger sur la pertinence de la métaphore en ellemême comme outil de pensée (Chaney, 2002 ; Stock, 2008). L'analogie entre art et vie sociale 
ne va pourtant pas de soi, l'art n'étant qu'un domaine restreint de cette vie sociale. L'utilisation de métaphores n'est pas un phénomène nouveau en géographie, ni dans les études touristiques. Le risque est de développer des raisonnements qui seraient en inadéquation avec le réel. Si on la prend au pied de la lettre, la métaphore de la performance pose effectivement problème. Penser les lieux comme des scènes, c'est imaginer des espaces limités qui fonctionneraient en vase clos. Ainsi, les travaux se sont portés prioritaire- ment sur des lieux facilement délimitables : restaurants, petits villages, sites et comptoirs touristiques, plages, avec le risque de se concentrer exclusivement sur les relations internes en négligeant les interactions avec l'extérieur. À l'inverse, la complexité des grandes métropoles, aux limites intra urbaines bien moins lisibles, n'a pas fait l'objet d'une attention suffisante.

Par ailleurs, penser les êtres humains comme des artistes mène à une appréhension biaisée des individus. Deux conséquences opposées sont à craindre : soit l'intentionnalité des acteurs est partout (ils donnent une certaine image d'eux-mêmes, trompent), comme l'artiste qui a conscience de produire de l'art, soit l'intention n'est nulle part, les individus ne faisant que répéter des rôles appris, à l'image de l'interprète. À moins qu'on ne s'attache, sur le mode d'une troisième voix, à la figure du performeur qui, pour avoir acquis des automatismes par l'apprentissage d'une technique, est ensuite capable des improvisations les plus innovantes...
(I) La géographie humaniste est parfois accusée d'être trop centrée sur l'intériorité du sujet et pas assez sur la matérialité des situations spatiales et sur la corporéité du rapport au monde (voir par exemple Nash, 2000).

(2) La Speech act theory, dont l'auteur le plus couramment cité est John Austin, est une théorie linguistique qui désigne la "performativité" comme la "propriété de la parole d'agir sur le monde et de le transformer" (Mondada, 2003). Voir Austin, 1970 (3) Le terme "envahissant" est employé ici pour conserver la connotation qui apparait en anglais dans le texte de Nigel Thrift à travers le terme "pervasive": "performance is, at this moment, one of the most pervasive metaphors in the human sciences" (Thrift, 2000b, p. 225).

(4) Une recherche par mots-clefs ("performance" et "performativity" dans l'ensemble du texte des articles) a été effectuée dans trois revues anglo-saxonnes emblématiques de la géographie humaine : Environment and Planning D : Society and Space; Progress in Human Geography; Annals of the Association of American Geographers. La revue Gender, Place and Culture a également fait l'objet de l'attention de l'auteur, comme espace de débat originel pour la métaphore de la performance en géographie.

(5) Bien que la question soit du plus grand intérêt, les travaux propres de l'auteur ne lui permettent pas, à ce stade de la réflexion, de trancher cette question, qu'elle laisse donc volontairement ouverte.

(6) Venise fournit un terrain particulièrement stimulant pour entamer cette réflexion. La mise en scène de l'espace urbain à des fins de séduction, notamment touristique, soit, si l'on adopte le vocabulaire de Mac Cannell, la production du front stage, est une construction longue et toujours en mouvement (voir notamment Davis, Marvin, 2004). Pour une lecture critique de la touristicité de l'espace urbain vénitien, voir Minca, Oakes, 2006b, où les deux chercheurs présentent leurs regards croisés sur une visite en commun de la ville. Pour une réflexion sur la manière dont les résidents vénitiens pratiquent au quotidien à la fois front stages et back stages, voir l'enquête menée par Quinn, 2007.

(7) Voir une proposition de l'auteur: Amandine CHAPUIS, "Amsterdam's back stage seductions, a geographical approach", communication du colloque Tourism and seduction of différence, Lisbonne, septembre 2010 .

(8) Le terme "re-création" est employé ici en référence au terme "recréation" issu du travail de l'Équipe Mit : "concept permettant de synthétiser un grand nombre de pratiques de rupture vis-à-vis des pratiques routinières, aboutissant à un relâchement plus ou moins contrôlé de l'auto contention des émotions (relâchement, défoulement, permissivité, etc., liés à une mise à distance du quotidien)" (Équipe Mit, 2005, p. 34I). Un tiret a cependant été ajouté pour attirer l'attention du lecteur sur cet emploi peu courant

(9) Voir une proposition de l'auteur: Amandine CHAPUIS, "Mélissa, Ninon, Jean-Philippe et Isma: expérimenter Amsterdam à 20 ans. Performances touristiques initiatiques et citadinités", communication aux Journées de la commission Approche culturelle en géographie de l'Union géographique internationale, Sion, juin 2010 


\section{RÉFÉRENCES BIBLIOGRAPHIQUES}

Hazel ANDREWs, "Feeling at home : embodying Britishness in a Spanish charter tourist ressort", Tourist Studies, vol. 5, 2005

John Langshaw Austin, Quand dire, c'est faire, Seuil, 1970 (| re édition 1962).

Jørgen Ole B/ERENHOLDT, Michael HaLdRUP, Jonas LARSEN et John URRY (dir.), Performing tourist places, Ashgate, 2004

\section{Adrian R. BAILEY, David C. HARVEY et}

Catherine BRACE, "Disciplining youthful methodist bodies in nineteenth-century", Annals of the Association of American Geographers, vol. 97, n I, 2007.

David BeLL, Jon BINNIE, Julia CREAM et Gill VALENTINE, "All hyped up and no place to go", Gender, Place and Culture, vol. I, 1994. Claudia BELL et John LYALL, "The accelerated sublime: thrill-seeking adventure heroes in the commodified landscape", dans Simon

Coleman et Mike Crang (dir.), Tourism, between Place and Performance, Berghahn Books, 2002.

Rachele BORGHI, Dinamiche coloniali e postcoIoniali nell'analisi dello spazio urbano di

Marrakech, Guéliz, thèse de doctorat en géographie, université Ca' Foscari di Venezia, 2006.

Judith BUTLER, Trouble dans le genre. Le féminisme et la subversion de l'identité, La Découverte, 2005, (I 're édition en anglais, Routledge, 1990).

Sarah G. CANT, "The tug of danger with the magnetism of mystery. Descents into the comprehensive, poetic-sensuous appeal of caves"', Tourist Studies, vol. 3, 2003.

Michel de Certeau, L'Invention du quotidien, t. I Arts de faire, coll. Folio, Gallimard, 1990 (Ire édition 1980).

David CHANEY, "The power of metaphors in tourism theory", dans Simon ColEMAN et
Mike CRANG (dir.), Tourism, between Place and Performance, Berghahn Books, 2002.

Christine Chivallon, “La géographie britannique et ses diagnostiques sur l'époque postmoderne", Cahiers de géographie du Québec vol. $43,118,1999$

Vincent CoËFFÉ, "Le tourisme, fabrique d'urbanité. Matériaux pour une théorie de l'urbain", Mondes du tourisme, 2, 2010.

Vincent CoËFfÉ, "L'urbanité cachée du rivage touristique", dans Philippe DuHAMEL et Rémy KNAFOU (dir.), Mondes urbains du tourisme, Belin, 2007.

Simon Coleman, Mike Crang (dir.), Tourism, between place and performance, Berghahn Books, 2002a.

Simon Coleman, Mike Crang "Grounded tourists, travelling theory", dans Simon

Coleman et Mike Crang (dir.), Tourism, between Place and Performance, Berghahn Books, $2002 b$.

Dwight CONQUergood, "Poetics, play, process, and power : the performative turn in anthropology", Text \& Performance Quarterly, vol. I, 1989

Jeremy W. CRAMPTON, "Cartography: maps 2.0", Progress in Human Geography, vol. 33, 2009.

Philip CRANG, "It's showtime: on the workplace geographies of display in a restaurant in southeast England", Environment and Planning D : Society and Space, vol. 12 (6), 1994.

Mike CrANG, "Circulation and emplacement : the hollowed-out performance of tourism", dans Claudio MincA et Tim OAKES (dir.), Travels in Paradox: Remapping Tourism, Rowman \& Littlefield Publishers, 2006.

David Crouch et Luke Desforges, "The sensuous in the tourist encounter : introduction : the power of the body in tourist stu- dies", Tourist Studies, vol. 3, n I, 2003

Robert C. DAVIS et Garry R. MARVIN, Venice, the Tourist Maze: a Cultural Critique of the World's Most Touristed City, University of California Press, 2004

Anne DOQuet, "'Se montrer Dogon. Les mises en scène de l'identité ethnique",

Ethnologies comparées, n 5, 2002.

Tim EDENSOR, "Sensing tourist spaces", dans Claudio Minca et Tim OAKES (dir.), Travels in Paradox : Remapping Tourism, Rowman \& Littlefield Publishers, 2006

Tim EdENSOR, "Performing tourism, staging tourism", Tourist Studies, vol. I, n I, 200।. Tim EDENSOR, Tourists at the Taj. Performance and Meaning at a Symbolic Site, Routledge, 1998.

ÉQUIPE MIT, Tourismes 2. Moments de lieux, coll. Mappemonde, Belin, 2005.

Erving GoffMAN, La Mise en scène de la vie quotidienne, t. I La présentation de soi, Les éditions de Minuit, 1973 (I re édition 1956).

Nicky Gregson et Gillian Rose, "Taking Butler elsewhere: performativities, spatialities and subjectivities", Environment and Planning D : Society and Space, vol. 18, 2000 Michael HALDRUP et Jonas LARSEN, "The family gaze", Tourist Studies, vol. 3.

Steven HoElSCHER, "Making Place, Making Race : Performances of Whiteness in the Jim Crow South", Annals of the Association of American Geographers, vol. 93, 3, 2003

Louise Holt, "Embodied social capital and geographic perspectives : performing the habitus", Progress in Human Geography, vol. 32, 2008.

Jessica JACOBS, "Tourist places and negociating modernity : European women and romance in the Sinai", dans Claudio MINCA et Tim OAKes (dir.), Travels in Paradox: Remapping Tourism, Rowman \& Littlefield Publishers, 2006 
Mark JAYNE, Gill VALENTINE et

Sarah L. HolzowaY, "Geographies of alcohol, drinking and drunkenness : a review of progress", Progress in Human Geography, vol. 32, 2008.

Rémy KNAFOU, "La transformation des lieux anciennement touristiques", Méditerranée, 3, 1996.

\section{Alan LATHAM, Derek P. McCORMACK, \\ "Moving cities : rethinking the materialities of urban geographies", Progress in Human Geography, vol. 28, 2004.}

Loretta LEES, "Rematerializing geography : The 'new' urban geography', Progress in Human Geography, vol. 26, 2002.

Jo LITTLE, Michael LeYSHON, "Embodied rural geographies : Developing research agendas", Progress in Human Geography, vol. 27, 2003.

Orvar Löfgren, On Holiday, a History of Vacationing, University of California Press, 1999.

Robyn LONGHURST, “ 'Corporeographies' of pregnancy : 'bikini babes'", Environment and Planning D : Society and Space, vol. 18, 2000.

Dean MacCanNelL, "Tourist agency", Tourist Studies, vol. I, 200I.

Dean MacCannell, The Tourist. A New Theory of the Leisure Class, University of California Press, 1999 (Ire édition 1976).

Linda MACDOWELL, "Thinking through work : complex inequalities, constructions of difference and trans-national migrants", Progress in Human Geography, vol. 32, 2008.

Claudio MiNCA, "The Island : work, tourism and the biopolitical", Tourist Studies, vol. 9 ,

2009.

Claudio MincA et Tim OAKES (DIR.), Travels in Paradox : Remapping Tourism, Rowman \& Littlefield Publishers, 2006a.

Claudio MinCA et Tim OAKES, "Traveling paradoxes", dans Claudio MiNCA et Tim

OAKES (dir.), Travels in Paradox: Remapping
Tourism, Rowman \& Littlefield Publishers, 2006.

Lorenza MoNDADA, "Performativité", dans Jacques LÉVY et Michel LussAuLt (dir.), Dictionnaire de la géographie et de l'espace des sociétés, Belin, 2003.

Catherine NASH, "Performativity in practice : some recent work in cultural geography", Progress in Human Geography, vol. 24(4), 2000.

Mark Neumann, "Making the scene : the poetics and performances of displacement at the Grand Canyon", dans Simon Coleman et Mike Crang (dir.), Tourism, between Place and Performance, Berghahn Books, 2002.

\section{Niels Kayser NielSEN, "New Year in}

Nämpnäs : on nationalism and sensuous holidays in Finland"', Tourist Studies, vol. 3, 2003.

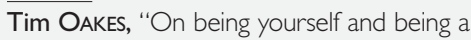
tourist", dans Claudio MINCA et Tim OAKES (dir.), Travels in Paradox: Remapping Tourism, Rowman \& Littlefield Publishers, 2006. Ruth PANelLI, "Social geographies: encounters with Indigenous and more-than-

White/Anglo geographies", Progress in Human Geography, vol. 32, 2008.

Chris Perkins, "Cartography - cultures of mapping: power in practice", Progress in Human Geography, vol. 28, 2004.

Geraldine PRATT, "Performance", dans Ron JOHNSTON, Derek GREGORY, Geraldine Pratt, Michael WatTs et Sarah Whatmore (dir.), The Dictionary of Human Geography, Blackwell, 2009.

Bernadette QUINN, “Performing tourism, venetian residents in focus", Annals of Tourism Research, vol. 34, 2007.

Richard SCHECHNER, Performance Studies. An Introduction, Routledge, 2002.

Matthew SPARKE, "Political geography. Political geographies of globalization III : résistance", Progress in Human Geography, vol. 32, 2008.
Jean-François STASZAK, "Qu'est ce que l'exotisme?"', Le Globe, tome 148, 2008.

Mathis STоск, "Penser géographiquement", dans Philippe MarTıN (dir.), "Demain la géographie", Géopoint, groupe Dupont et UMR Espace, Université d'Avignon, 2008.

Mathis Sтоск, Mobilités géographiques et pratiques des lieux. Étude théorico-empirique à travers deux lieux touristiques anciennement constitués : Brighton \& Hove (Royaume-Uni) et Garmisch-Partenkirchen (Allemagne), thèse de géographie (sous la direction de Rémy Knafou), Université de Paris 7 - Denis

Diderot, 200I.

Nigel THRIFT, "Performance", dans Ron JOHNSTON, Derek GREGORY, Geraldine Pratt, Michael WATtS and Sarah WHATMORE (dir.), The Dictionary of Human Geography, Blackwell, 2000a.

Nigel THRIFT, "Afterwords", Environment and Planning D: Society and Space, vol. 18, 2000b.

Nigel THRIFT et John-David DeWsBURY, "Dead geographies - and how to make them live", Environment and Planning D: Society and Space, vol. 18, 2000.

Hazel TUCKER, "Narratives of place and self : differing experiences of package coach tours in New Zealand", Tourist Studies, vol. 5, 2005. John URRY, The Tourist Gaze, Sage, 1990.

Adam WEAVER, "Interactive service work and performative metaphors. The case of the cruise industry", Tourist Studies, vol. 5, 2005. 